\title{
Resonance-like electrical control of electron spin for microwave measurement *
}

\author{
B. A. Glavin \\ V.E. Lashkar'ov Institute of Semiconductor Physics, \\ Pr. Nauki 41, Kiev 03028, Ukraine \\ K. W. Kim \\ Department of Electrical and Computer Engineering, \\ North Carolina State University, Raleigh, NC 27695-7911, USA
}

\begin{abstract}
We demonstrate that the spin-polarized electron current can interact with a microwave electric field in a resonant manner. The spin-orbit interaction gives rise to an effective magnetic field proportional to the electric current. In the presence of both dc and ac electric field components, electron spin resonance occurs if the ac frequency matches with the spin precession frequency that is controlled by the dc field. In a device consisting of two spin-polarized contacts connected by a two-dimensional channel, this mechanism allows electrically tuned detection of the ac signal frequency and amplitude. For GaAs, such detection is effective in the frequency domain around tens of gigahertz.
\end{abstract}

PACS numbers: 72.25.Dc, 76.30.-v, 73.63.Kv

\footnotetext{
* The following article has been submitted to Applied Physics Letters. After it is published, it will be found at http://apl.aip.org
} 
During the last decade, there has been considerable interest in spin-based applications for data storage and processing. One of the widely discussed designs is the so-called spin fieldeffect transistor $\stackrel{1}{\underline{1}}$ In this device, the source and drain contacts are spin-polarized (which can be achieved, for example, in a diluted magnetic semiconductor in the ferromagnetic phase). Spin-polarized electrons injected from the source contact are transferred to the drain through a quantum well $(\mathrm{QW})$ channel. In the course of this transfer, the electron spin precesses due to the spin splitting of QW energy band spectrum. When the spin splitting is mainly due to the Bychkov-Rashba mechanism,, 2 the magnitude of splitting and, consequently, the frequency of spin precession can be modified by the transverse electric field controlled by the gate voltage. Therefore, both the electron spin polarization and current at the drain are controlled by the gate voltage.

In this letter, we propose a different, gate-less scheme of nonequilibrium spin control in a similar device, whose schematics is shown in the insert of Fig. 1. The proposed method relies on the interaction of spin-polarized electrons with a microwave electric field. Although this interaction looks similar to the electric-dipole resonance, $\frac{3}{3}$ the main difference is that the effective splitting in our case is induced by the electric current rather than the magnetic field and, therefore, allows purely electrical tuning. We derive criteria for effective spin control and analyze them for various semiconductor materials. Our results suggest that the proposed device structure can be used to determine the frequency, amplitude, and polarization of microwave radiation with potential advantages over the existing approaches. $\underline{\underline{4}}$

Let us start with the consideration of spin dynamics in a $\mathrm{QW}$ in the presence of electric field $\boldsymbol{E}$. The electron Hamiltonian is

$$
H=\frac{\hbar^{2} k^{2}}{2 m}+\frac{\hbar}{2} \boldsymbol{\sigma} \boldsymbol{\Omega}+\hat{V},
$$

where $k$ is the electron momentum and $m$ is the electron effective mass. The second term describes the spin splitting of the electron spectrum in a $k$-dependent effective magnetic field and the third term corresponds to the electron interaction with the electric field. Two contributions to $\Omega$ proportional to the electron wavevector can be important. The bulk-induced contribution $\Omega_{b}$ is due to the absence of the inversion center in the crystal constituting the QW, while the structure-induced contribution $\Omega_{s}$ arises from the asymmetry of the QW structure. $\stackrel{2}{2}$ For the particular case of a cubic QW grown along the (001) direction, we have $\boldsymbol{\Omega}_{b}=\alpha\left(k_{x} ;-k_{y} ; 0\right)$ and $\boldsymbol{\Omega}_{s}=\beta\left(k_{y} ;-k_{x} ; 0\right)$. Here, $\alpha$ and $\beta$ are the material and structure- 
dependent parameters. Since $\Omega$ is an odd function of the electron wavevector, its average value is zero if there is no current in the system. For a finite current, however, it becomes non-zero and the electrons experience the influence of average effective magnetic field. $\frac{5}{\cdot}$ Here, we make use of the fact that if both dc and ac components of the electric current are present, they can give rise to electron spin resonance even in the absence of ac and dc magnetic fields.

It is convenient to treat this resonance phenomenon by using a hydrodynamic spin transport model. If the electron density is spatially uniform, the evolution of the average electron spin $\boldsymbol{S}$ can be described by the following equation: $\underline{\underline{6}}$

$$
\frac{\partial \boldsymbol{S}}{\partial t}=\overline{\boldsymbol{\omega}} \times \boldsymbol{S}-v_{x} \frac{\partial \boldsymbol{S}}{\partial x}+D \frac{\partial^{2} \boldsymbol{S}}{\partial x^{2}}-\hat{\gamma} \boldsymbol{S} .
$$

Here, we assume that the electric current is parallel to the $x$ axis; $v_{x}$ is the $x$-component of the electron drift velocity, $D$ is the diffusion coefficient, $\overline{\boldsymbol{\omega}}$ is the average effective magnetic field in units of frequency, and $\hat{\gamma}$ is the relaxation matrix. Equation (22) is applicable if all the characteristic times related to spin evolution are much longer than the electron orbital relaxation times or, in other words, when the spin-orbit (SO) interaction is weak. To be specific, we consider in the following the case when the bulk-induced contribution is the most important [i.e., $\left.\overline{\boldsymbol{\omega}}=\alpha\left(m v_{x} / \hbar ;-m v_{y} / \hbar ; 0\right)\right]$ and spin relaxation is due to the D'yakonov-Perel' mechanism. $?$ These conditions correspond, in particular, to GaAs/AlGaAs QWs. In this case, the nonzero components of the relaxation matrix are $\gamma_{x x}=\gamma_{y y}=\gamma$, $\gamma_{z z}=2 \gamma, \gamma \approx(\Omega(\bar{k}))^{2} \tau$, where $\bar{k}$ is the characteristic electron wavevector and $\tau$ is the electron relaxation time. $\underline{\underline{7}}$

In the following, we neglect spin diffusion (the justification will be discussed explicitly below) and assume that the dc (ac) field is parallel to the $x(y)$ axis. Under these conditions, $\overline{\boldsymbol{\omega}}=\boldsymbol{\omega}_{0}+\boldsymbol{\omega}_{1} \cos \omega t$, where $\boldsymbol{\omega}_{0}=\alpha\left(m v_{0} / \hbar ; 0 ; 0\right), \boldsymbol{\omega}_{1}=\alpha\left(0 ;-m v_{1} / \hbar ; 0\right), v_{0}$ and $v_{1} \cos \omega t$ are the dc and ac drift velocities, respectively, and $\omega$ is the frequency of the ac signal. For convenience, we define $\alpha m v_{0} / \hbar$ and $\alpha m v_{1} / \hbar$ as $\omega_{0}$ and $\omega_{1}$, respectively. The growth direction is chosen to be the $z[(001)]$ axis.

The solution of Eq. (21) with $D=0$ can be sought in the form $\boldsymbol{S}(x, t)=\boldsymbol{S}\left(x-v_{0} t, t\right)$, which transforms it into the ordinary differential equations similar to the Bloch equations for spin resonance. $\underline{\underline{8}}$ If the initial spin state near the source $(x=0)$ is $\boldsymbol{S}_{0}$, then its value at the drain contact $(x=L)$ can be found as $\left.\boldsymbol{S}\right|_{t=L / v_{0}}$. Of course, the spin state at the drain depends on the phase of ac field. For simplicity, we adopt the value averaged over this 
parameter.

In the frame of reference rotating around $\boldsymbol{\omega}_{0}$ with the frequency $\omega$, it is well known that electron spin dynamics can be described as precessing around the vector $\left(\omega_{0}-\omega, \omega_{1} / 2 ; 0\right)$ if no relaxation is present (the factor $1 / 2$ is due to the fact that we consider a linearly polarized ac field). Effective resonance control of the spin polarization occurs if the spin at the source is parallel to $\boldsymbol{\omega}_{0}$. If there is no resonance of $\omega_{0}$ and $\omega$, then the spin remains practically unchanged during the transfer to the drain. In contrast, under the resonance condition $\left(\omega \approx \omega_{0}\right)$, spin precesses around $\boldsymbol{\omega}_{1}$ with the frequency $\omega_{1} / 2$ in the rotating frame. In particular, if $\omega_{1} L / v_{0}=2 \pi$, the spin flips during the transfer through the QW, which can be registered by the drain current. This clearly provides a possibility to probe the frequency of the microwave signal by a simple electrical tuning of the system into resonance.

Let us discuss the necessary conditions for supporting effective resonance in the structure. Presumably, spin diffusion makes the resonance weaker. Therefore, the drift term in Eq. (2) must exceed the diffusion term. The characteristic length of spin change is $v_{0} / \omega_{0}$. Using the Einstein relation for the diffusion coefficient, we find that the diffusion can be neglected if $(\Omega(\bar{k}))^{2} \tau_{p} / \omega_{0} \ll 1$, where $\tau_{p}$ is the electron momentum relaxation time which determines the mobility. This condition means that it is desirable to apply a sufficiently strong de electric field in a low-mobility sample. The second condition requires that many precession cycles must occur during the spin transfer through the QW. Therefore the QW length must be $L \gg L_{0} \equiv \hbar /(m \alpha)$, where $L_{0}$ depends only on the material parameters. Finally, the spin decay during the transfer must be negligible: $\left(\gamma / \omega_{0}\right)\left(L / L_{0}\right) \ll 1$.

We can estimate the amplitude of the ac field $E^{*}$ required for spin flip. Using the estimate for spin relaxation rate, we obtain $e E^{*} \gg \hbar \alpha \bar{k}^{2}\left(\tau / \tau_{p}\right)$. Clearly, the sensitivity of the device is better for materials with a weaker SO interaction. This is not surprising since the SO coupling controls both spin precession and relaxation. Note that for a wide range of structure parameters, $\tau$ is determined by the electron-electron collisions ${ }^{9,10}$ and $\tau / \tau_{p} \ll 1$.

Figure 1 shows the numerical results for the ratio $S_{d} / S_{s}$, where $S_{s}$ and $S_{d}$ are the $x$ component of the spin at the source and drain contacts, respectively. In the following, we will refer to this ratio as spin response. The calculation is performed for the microwave radiation frequency of $50 \mathrm{GHz}$ in a GaAs/AlGaAs QW with $\alpha=5 \times 10^{5} \mathrm{~cm} / \mathrm{s}, \gamma=6 \times 10^{9} \mathrm{~s}^{-1}$, the mobility $\mu=2500 \mathrm{~cm}^{2} / V s, L=10 \mu \mathrm{m}$, and several values of ac field $E_{1}$. The parameters used in this study are close to those of the GaAs QW measured at room temperature.11 
As can be seen from the figure, the resonance in the spin response appears for a relatively large $E_{1}$ (note that for a electromagnetic plane wave, $E_{1}=1 \mathrm{kV} / \mathrm{cm}$ corresponds roughly to the energy flux of $1.5 \mathrm{~kW} / \mathrm{cm}^{2}$ in vacuum). For a moderately high $E_{1}$, the resonance dip of the spin response increases with $E_{1}$. This corresponds to the gradual increase of the spin rotation angle up to a complete flip. The further increase of $E_{1}$ leads to a nonresonant spin response character due to multiple spin rotation cycles during the electron transfer between the source and the drain. However, even for a strong field the resonant character of spin response can be restored by changing the device orientation with respect to the acfield polarization. This is because the spin rotation is mainly due to the ac-field component perpendicular to the dc field. Such a behavior is illustrated in Fig. 2, where the spin response at $E_{1}=1.5 \mathrm{kV} / \mathrm{cm}$ is plotted for different angles $\phi$ between the ac and dc fields; clearly, the $\phi=\pi / 8$ case recovers a pronounced resonance pattern. In the inset, we show the dependence of spin response on $E_{1}$ under the resonance condition $\left(\omega=\omega_{0}\right)$. As expected, a periodic dependence is observed, which corresponds to the periodic change in the phase of the rotating spin at the drain. If the dc and ac fields are parallel, the spin response becomes a smooth function of de field.

The observed spin response properties suggest that the proposed device structure can be used for detecting the frequency, amplitude, and polarization of a microwave radiation. Under a measurement set-up, it is possible first to detect the polarization by finding device orientation characterized by the absence of resonant features in the spin response (namely, the orientation that the device dc field aligns with the microwave ac field). Then, repositioning the device orientation for clear resonant features, one can determine the frequency by the location of resonance and the amplitude by its depth. Note that it is possible to determine if the radiation is circularly polarized by the change of the spin response when the dc bias is reversed.

Let us stress once more that scanning over the frequency range occurs by simple variation of the dc field. The alternative methods of microwave frequency measurements require either the mechanical tuning of microwave cavity eigenfrequency or the use of a superheterodyne technique, followed by intensity measurements made by the devices insensitive to the frequency, such as thermocouples, bolometers, or Schottky diodes. ${ }^{4}$ Since these approaches allow detection of much weaker radiation than the considered above, the proposed resonance method is most suitable for high-power applications where measurements require the use of 
massive calorimetric devices or attenuation techniques. $\stackrel{4}{-}$ It is also important to note that the proposed device practically does not absorb power; in the structure considered with $E_{1}=1 \mathrm{kV} / \mathrm{cm}$, the absorbed ac power density is about $8 \mathrm{~W} / \mathrm{cm}^{2}$ for an electron density of $4 \times 10^{10} \mathrm{~cm}^{-2}$, which is more than two orders of magnitude less than the microwave radiation energy flux. This means that the device effectively does not disturb propagation of radiation.

Furthermore, the sensitivity of the proposed scheme may improve by proper choice of the material system. Recently, weak SO interaction was claimed in bulk GaN and GaN-based QWs ${ }^{12}$ with $\beta$ as low as $10^{5} \mathrm{~cm} / \mathrm{s}$. According to the estimate, this provides a possibility to reduce the radiation power requirement by more than an order of magnitude compared to the GaAs-based counterpart. Alternatively, the measurement can be achieved by the socalled electrically detected magnetic resonance (EDMR) (see, for example, Refs. 13 and 14). In this case, the frequency is determined by the resonant change of differential conductivity due to electron spin polarization and does not require spin-polarized electrodes. Since the physical basis of EDMR remains unclear, it is difficult to estimate the degree of conductivity variation for the considered case of electrically-controlled resonance.

In conclusion, we predict an effective resonance control of nonequilibrium electron spin by a microwave electric field. Based on this phenomenon, it is possible to achieve efficient electrically tuned measurement of frequency, polarization, and amplitude of the microwave radiation.

The authors would like to thank V.I. Sheka for fruitful discussions. The work was supported by the Defence Advanced Research Projects Agency and the CRDF (grant UE22439-KV-02). 
1 S. Datta and B. Das, Appl. Phys. Lett. 56, 665 (1990).

2 Yu. A. Bychkov and E. I. Rashba, Pis'ma Zh. Eksp. Teor. Fiz. 39, 66 (1984) [Sov. Phys. JETP Lett. 39, 78 (1984)].

3 E. I. Rashba and V. I. Sheka, in Landau Level Spectroscopy, edited by G. Landwehr and E. I. Rashba (Elsevier, New York, 1991).

4 G. H. Bryant, Principles of Microwave Measurments (Peter Peregrinus, London, 1993).

5 A. G. Aronov and Yu. B. Lyanda-Geller, Pis'ma Zh. Eksp. Teor. Fiz. 50398 (1989) [JETP Lett. 50, 431 (1989)]; V. M. Edelstein, Solid State Commun. 73, 233 (1990); L. I. Magarill, A. V. Chaplik, and M. V. Entin, Fiz. Techn. Polupr. 35, 1128 (2001) [Semicond. 35, 1081 (2001)].

6 S. Saikin, cond-matt/0311221.

7 M. I. D'yakonov and V. I. Perel, Fiz. Tverd. Tela 13, 3581 (1971) [Sov. Phys. Solid State 13, 3023 (1972); M. I. D’yakonov and V. Yu. Kachorovskii, Fiz. Techn. Polupr. 20, 178 (1986) [Sov. Phys. Semicond. 20, 110 (1986)].

8 A. Abragam, The Principles of Nuclear Magnetism (Clarendon, Oxford, 1961).

9 M. M. Glazov and E. L. Ivchenko, Pisma Zh. Eksp. Teor. Fiz. 75, 476 (2002) [JETP Lett. 75, $403(2002)]$.

10 R. T. Harley, M. A. Brand, A. Malinowski, O. Z. Karimov, P. A. Marsden, A. J. Shields, D. Sanvitto, D. A. Ritchie, and M. Y. Simmons, Physica E 17, 324 (2003).

11 Y. Ohno, R. Terauchi, T. Adachi, F. Matsukura, and H. Ohno, Physica E 6, 817 (2000).

12 S. Krishnamurthy, M. van Schilfgaarde, and N. Newman, Appl. Phys. Lett. 83, 1761 (2003); V.I. Litvinov, Phys. Rev. B 68, 155314 (2003).

13 M. Guèron and I. Solomon, Phys. Rev. Lett. 15, 667, (1965); A. Honig, Phys. Rev. Lett. 17, 186 (1966); 17, 188 (1966).

14 C.F.O. Graef, S. Brandt, M. Stutzmann, M. Holzmann, G. Abstreiter, and F. Schäffler, Phys. Rev. B 59, 13242 (1999). 


\section{Figure Captions}

FIG. 1. Spin response as a function of effective precession frequency for a GaAs/AlGaAs QW for several values of microwave field. The calculations are done for the microwave frequency of $50 \mathrm{GHz}$. The insert shows the schematics of the proposed device. The black regions represent spin-polarized contacts connected by the QW.

FIG. 2. Spin response for the amplitude of ac field $E_{1}=1.5 \mathrm{kV} / \mathrm{cm}$ and different angles $\phi$ between the ac and dc fields. The insert shows the dependence of spin response on $E_{1}$ in resonance for two values of $\phi$. 


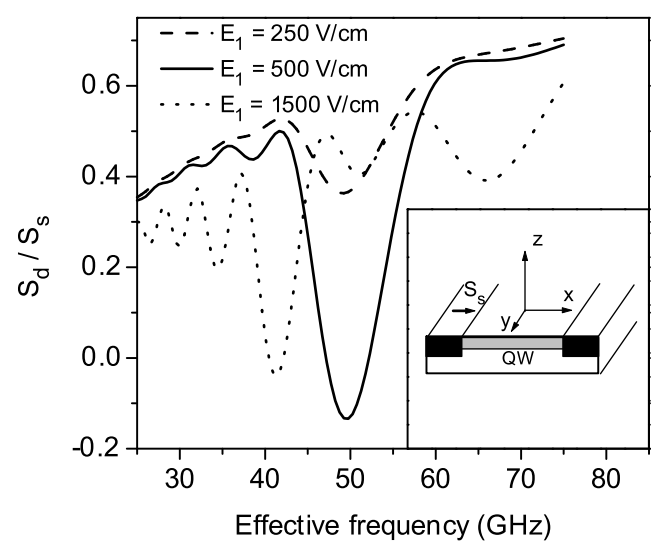

Fig. 1. 


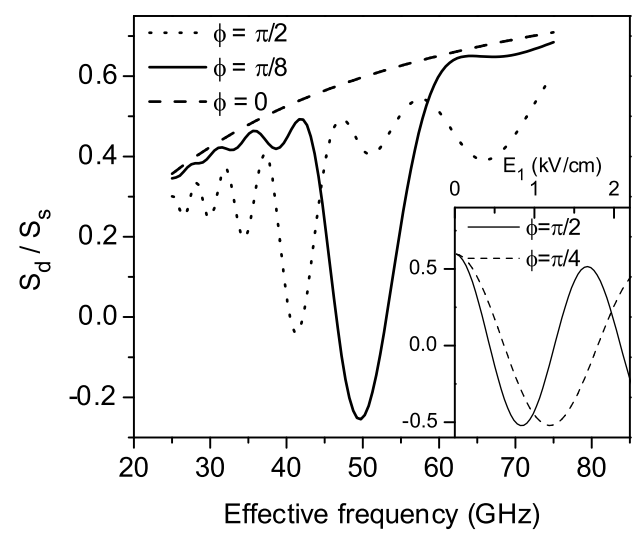

Fig. 2. 